\title{
Metodologia alternativa de medida da densidade de meios porosos em laboratórios didáticos de física
}

\author{
Alternative methodology of porous media bulk density measurement in didactic physics laboratories \\ Mario A. Camargo*1@, Luiz F. Pires ${ }^{2}$, André M. Brinatti², Sérgio C. Saab ${ }^{20}$ \\ ${ }^{1}$ Instituto Federal de Santa Catarina, Campus Canoinhas, SC, Brasil \\ ${ }^{2}$ Universidade Estadual de Ponta Grossa, Departamento de Física, PR, Brasil
}

Recebido em 27 de julho de 2020. Revisado em 21 de agosto de 2020. Aceito em 22 de setembro de 2020.

\begin{abstract}
Quando se estuda a qualidade física de meios porosos como o solo uma propriedade de bastante relevância é a sua densidade, $\rho_{s}$. As medidas de $\rho_{s}$ são importantes devido à influência desta propriedade em outras deste sistema poroso (e.g., porosidade, umidade volumétrica, armazenamento de água, etc). Neste trabalho propõe-se uma metodologia alternativa para a medida de $\rho_{s}$, chamada de método da sela a vácuo. A impermeabilização da amostra, contrário aos métodos tradicionais como torrão parafinado, é feita utilizando embalagem plástica com posterior evacuação do ar interno usando bomba de vácuo. Medidas de $\rho_{s}$ pelo método do torrão parafinado foram utilizadas como referência. Os resultados obtidos mostram que em relação ao método do torrão parafinado, $\rho_{s}$ medido pelo método da sela a vácuo é, em média, subestimado para todos os diferentes tipos de solos analisados. O método proposto é mais rápido e apresenta maior confiabilidade na impermeabilização que o método tradicional, além disso, permite reutilizar a amostra para novas medidas. O novo método ainda pode ser facilmente implementado em laboratórios de ensino de física para o ensino médio e para o ensino superior nos cursos de bacharelado, engenharias e agronomia. Por se tratar de um método que envolve pouco manuseio, rápido e de fácil implementação, torna-se ideal para o estudo não somente de $\rho_{s}$, mas também de conceitos envolvidos em sua medida como massa, volume, massa específica, peso, peso aparente e empuxo.
\end{abstract}

Palavras-chave: método da sela a vácuo, torrão parafinado, meio poroso, densidade do solo, ensino de física.

When the physical quality of porous media such as soil is studied one of the properties of great importance is its bulk density, $\rho_{s}$. Measurements of $\rho_{s}$ have great relevance due to its influence in other porous system properties (e.g. porosity, volumetric water content, water storage, etc.). In this paper is proposed an alternative methodology of $\rho_{s}$ measurement named vacuum-sealed method. Samples coating, contrary to the traditional methods such as the paraffin-coated, was carried out using a plastic packaging followed by the sample internal air evacuation through a vacuum pump device. The determination of the clod samples $\rho_{s}$ by the paraffin-coated method was utilized as a reference. The obtained results showed that compared to the paraffin-coated method, the average $\rho_{s}$ measured by the vacuum-sealed method presented underestimated values considering all the soils analyzed. The proposed method is faster and has greater coating reliability than the traditional method with the additional advantage of sample reuse for new measurements. The new method can also be easily implemented in teaching physics laboratories in high school grade and bachelor's, engineering, and environmental science courses. As a method that requires minimal sample preparation, easy to implement, and fast, it becomes ideal for the study not only of $\rho_{s}$ but also to the discussion of concepts involved in its measurement such as mass, volume, weight, apparent weight and buoyancy force.

Keywords: vacuum-sealed method, paraffin-coated clod, porous media, soil bulk density, physics teaching.

\section{Introdução}

Na área de física aplicada a meios porosos, em especial em estudos sobre a estrutura do solo, a sua densidade, $\rho_{s}$, representa um atributo físico de grande relevância devido a sua sensibilidade a mudanças em fatores naturais e antropogênicos [1. Por este motivo, $\rho_{s}$ é amplamente utilizada como parâmetro para avaliar a qualidade física deste sistema poroso, permitindo inferir sobre problemas ambientais tais como: compactação do solo, infiltração

\footnotetext{
*Endereço de correspondência: mario.camargo@ifsc.edu.br
}

de contaminantes, sequestro e liberação de gases do efeito estufa, entre outros [2 5].

Existem diversos métodos de medida de $\rho_{s}$, sendo classificados como diretos (anel volumétrico, torrão parafinado, escavação) e indiretos (atenuação de raios gama, tomografia computada, sonda de nêutrons-gama). Cada um destes métodos apresenta vantagens e desvantagens com relação a custos, condições de uso, tamanho de amostras, tempo de medida, etc [6, 7]. Um método frequentemente utilizado para amostras de formato irregular é o do torrão parafinado, no qual se impermeabiliza um agregado de solo com parafina fundida 
visando posterior obtenção de seu volume por imersão em água [8]. Entretanto, este método além de ser demorado, pode permitir a entrada de parafina fundida nos macroporos do agregado afetando as medidas de $\rho_{s}$ 9 11.

Neste trabalho é apresentada uma metodologia alternativa de medida de $\rho_{s}$ utilizando um sistema de sela a vácuo. A impermeabilização do agregado é feita usando embalagem plástica que assume sua forma externa, devido a evacuação do ar interno, pela ação de uma bomba de vácuo. Este novo método apresenta como vantagens: 1) a capacidade de determinar $\rho_{s}$ com maior agilidade, 2) a minimização dos efeitos do método experimental na aferição do volume do agregado e 3) possibilidade de reutilização das amostras em outras análises, devido ao caráter não destrutivo do método proposto.

O método apresenta ainda como diferencial a possibilidade de sua utilização em laboratórios dedicados ao ensino de física; por se tratar de um método limpo, pois diminui a possibilidade de quebra de agregados e poeira, rápido e de fácil implementação. Por meio dos procedimentos aqui propostos conceitos como massa, volume, massa específica, peso real, peso aparente e empuxo poderão ser trabalhados.

\section{Material e Métodos}

\subsection{Amostras de solo}

As amostras indeformadas utilizadas neste estudo foram coletadas na camada superficial do solo $(0-15 \mathrm{~cm})$ consistindo de agregados com volumes variando de aproximadamente 235 a $392 \mathrm{~cm}^{3}$. Quatro tipos diferentes de solos foram analisados: Latossolo Vermelho-Amarelo solos A e B, Nitossolo Vermelho - solo C, Latossolo Bruno - solo D e Cambissolo Háplico Alumínico - solo E [12]. O Latossolo Vermelho-Amarelo possuía duas classes texturais distintas e, por este motivo, foi classificado como dois solos. Um total de 4 amostras foram coletadas para cada tipo de solo totalizando 20 amostras. A Tabela 1 apresenta os valores das frações argila, silte e areia dos solos analisados.

\subsection{Preparação das amostras}

As amostras foram secadas em estufa a $40^{\circ} \mathrm{C}$ por 24 horas antes da realização das medidas. Após este

Tabela 1: Valores das frações argila, silte e areia dos solos analisados. Os nomes dos solos seguiram a nomenclatura do Sistema Brasileiro de Classificação do Solo - SiBCS [12].

\begin{tabular}{llccc}
\hline & & \multicolumn{3}{c}{$(\mathrm{g} / \mathrm{kg})$} \\
\cline { 3 - 5 } SOLO & \multicolumn{1}{c}{ Nome } & Argila & Silte & Areia \\
\hline A & Latossolo Vermelho-Amarelo & 397 & 105 & 498 \\
B & Latossolo Vermelho-Amarelo & 280 & 60 & 660 \\
C & Nitossolo Vermelho & 430 & 330 & 240 \\
D & Latossolo Bruno & 426 & 223 & 351 \\
E & Cambissolo Háplico Alumínico & 455 & 494 & 51 \\
\hline
\end{tabular}

período, aferiu-se a massa de solo de cada amostra e impermeabilizaram-se os agregados por meio de dois métodos: sela a vácuo e parafina fundida - torrão parafinado [8].

O material empregado na impermeabilização das amostras de solo pelo método da sela a vácuo foi uma embalagem plástica comumente usada na conservação de alimentos a vácuo (filme plástico composto por poliamida e polietileno sem proporções conhecidas). No método proposto a amostra é acondicionada dentro da embalagem plástica para posterior retirada do ar interno da embalagem (processo a vácuo), moldando a embalagem plástica ao formato do agregado de solo (Figura 17). Neste passo sugere-se utilizar um tamanho de embalagem plástica otimizado para acomodar a amostra minimizando gastos com este insumo.

Cabe ressaltar que todo o processo de impermeabilização pelo método da sela a vácuo é realizado pela máquina seladora a vácuo, ou seja, uma vez colocada a embalagem plástica com a amostra de solo acondicionada em seu interior, o acionamento da máquina a vácuo possibilita a impermeabilização do agregado em menos de 30 segundos.

Para o método do torrão parafinado, a impermeabilização se deu pela solidificação da parafina fundida na amostra após imersão completa (Figura 1 b). Para fundir a parafina utilizou-se uma chapa aquecedora com regulagem de temperatura em $65^{\circ} \mathrm{C}$. Após a fusão da parafina, imergiram-se as amostras por completo retirando-as rapidamente para que a parafina pudesse se solidificar a temperatura ambiente. Após este procedimento verificou-se a formação de uma camada impermeável no entorno dos agregados de solo. Quando foi verificado algum problema na camada solidificada de parafina, devido a rachaduras ou pequenos orifícios, todo o procedimento foi repetido visando a completa impermeabilização da amostra. Para facilitar este procedimento, os agregados foram mergulhados utilizandose barbantes fixados a eles (Figura 1b) 9 .

\subsection{Massa específica do plástico}

Devido à inexistência de informações precisas sobre a composição do plástico da embalagem, a determinação de sua massa específica, $\rho_{e}$, fez-se necessária. O procedimento empregado foi a medida da massa da embalagem plástica, $m_{e}$, em balança de precisão (Gehara AG200, resolução de $0,0001 \mathrm{~g}$ ) e, posteriormente, divisão pelo seu volume. Devido o pequeno valor de $m_{e}$, fez-se necessário neste trabalho o uso de uma balança de alta precisão, porém é valido ressaltar que o emprego de uma balança de menor precisão não inviabiliza a utilização do método proposto.

Calculou-se o volume da embalagem plástica multiplicando os valores de comprimento, largura e espessura. Os dois primeiros foram medidos com uma trena (resolução de $1 \mathrm{~mm}$ ) e o último com um micrômetro (resolução de $0,01 \mathrm{~mm})$. Para a massa específica da parafina, $\rho_{p}$, e da 


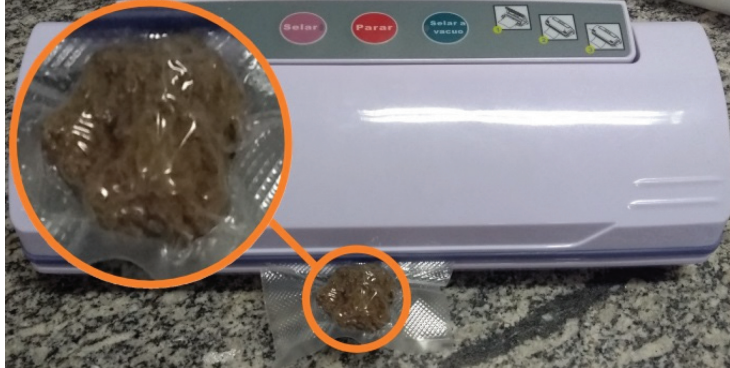

(a)

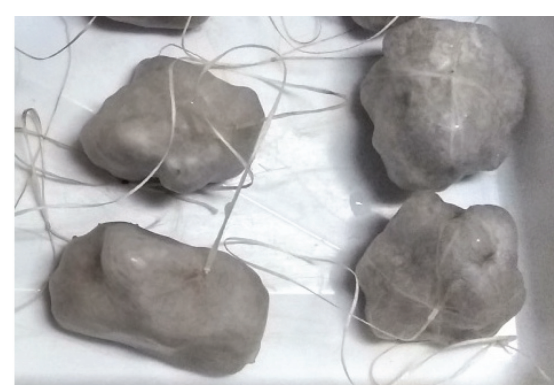

(b)

Figura 1: Amostras impermeabilizadas. (a) Método da sela a vácuo: máquina seladora a vácuo com agregado de solo sendo evacuado e impermeabilizado e (b) método da parafina fundida: agregados de solo amarrados com barbantes e revestidos por uma camada de parafina.

água destilada, $\rho_{l}$, foram utilizados valores encontrados na literatura [13.

\subsection{Densidade do solo}

A densidade do solo foi calculada pela equação (1):

$$
\rho_{s}=\frac{m_{s}}{V_{t}}
$$

sendo $m_{s}$ a massa de solo seco (secado em estufa a $105^{\circ} \mathrm{C}$ por $24 \mathrm{~h}$ ) e $V_{t}$ é o volume total da amostra.

A massa do solo seco, a massa da embalagem plástica e a massa da parafina, $m_{p}$, foram medidas no momento da preparação das amostras utilizando uma balança de precisão (Gehara AG200, resolução de 0,0001 g). O volume total foi determinado de maneira indireta utilizando um arranjo constituído de balança, béquer contendo água destilada com volume suficiente para completa imersão da amostra e suporte para suspensão da amostra (Figura 2).
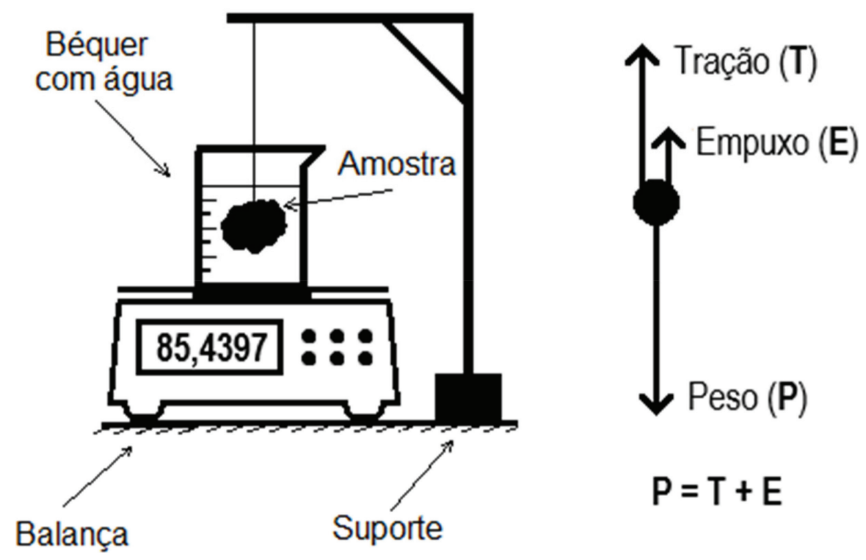

Figura 2: Desenho esquemático do arranjo experimental utilizado para determinar a massa do líquido deslocado pela amostra de solo impermeabilizado. Das forças que atuam sobre a amostra, apenas o empuxo exerce reação sobre a balança e esta, por sua vez, mostra o valor da massa de líquido deslocado pela amostra.
No procedimento experimental o agregado é completamente imerso em água destilada, de modo a não tocar no fundo ou na parede do béquer. A balança deve estar tarada (descontando a massa do conjunto béquer + água destilada) antes da imersão da amostra. A leitura da balança fornece o valor da massa de líquido deslocado, $m_{l}$, pela amostra do agregado impermeabilizado. O volume total é então calculado pela equação (2):

$$
V_{t}=\left(\frac{m_{l}}{\rho_{l}}\right)-\left(\frac{m_{i}}{\rho_{i}}\right)
$$

sendo $m_{i}$ a massa do impermeabilizante utilizado (embalagem plástica ou parafina fundida) e $\rho_{i}$ é a massa específica do impermeabilizante utilizado.

\section{Resultados e Discussões}

Para a avaliação do método da sela a vácuo poder ser utilizado como alternativo ao torrão parafinado, os dois métodos foram aplicados nas mesmas amostras. Este tipo de procedimento de comparação é interessante porque evita problemas de variabilidade espacial quando diferentes amostras são utilizadas [14]. Por exemplo, o trabalho de [15] traz uma análise detalhada da variabilidade espacial da estrutura interna de amostras de agregados de solo, demonstrando a importância de se considerar esta variabilidade em medidas de atributos físicos deste sistema poroso. Neste sentido, primeiramente impermeabilizaram-se as amostras pelo método da sela a vácuo e, posteriormente, com a parafina fundida, visando o mínimo de perturbação possível nas amostras.

A distribuição dos valores de $\rho_{s}$, obtidos para os diferentes solos estudados, estão apresentados na Figura 3. Verificou-se que os valores da densidade do solo medidos pelo método da sela a vácuo, $\rho_{s v}$, estão subestimados (a reta de ajuste está abaixo da reta 1:1 e o coeficiente angular da reta de ajuste é menor que a unidade) na comparação com os valores medidos pelo método do torrão parafinado, $\rho_{s t}$, considerado método de referência neste trabalho. Este comportamento foi verificado para todos 

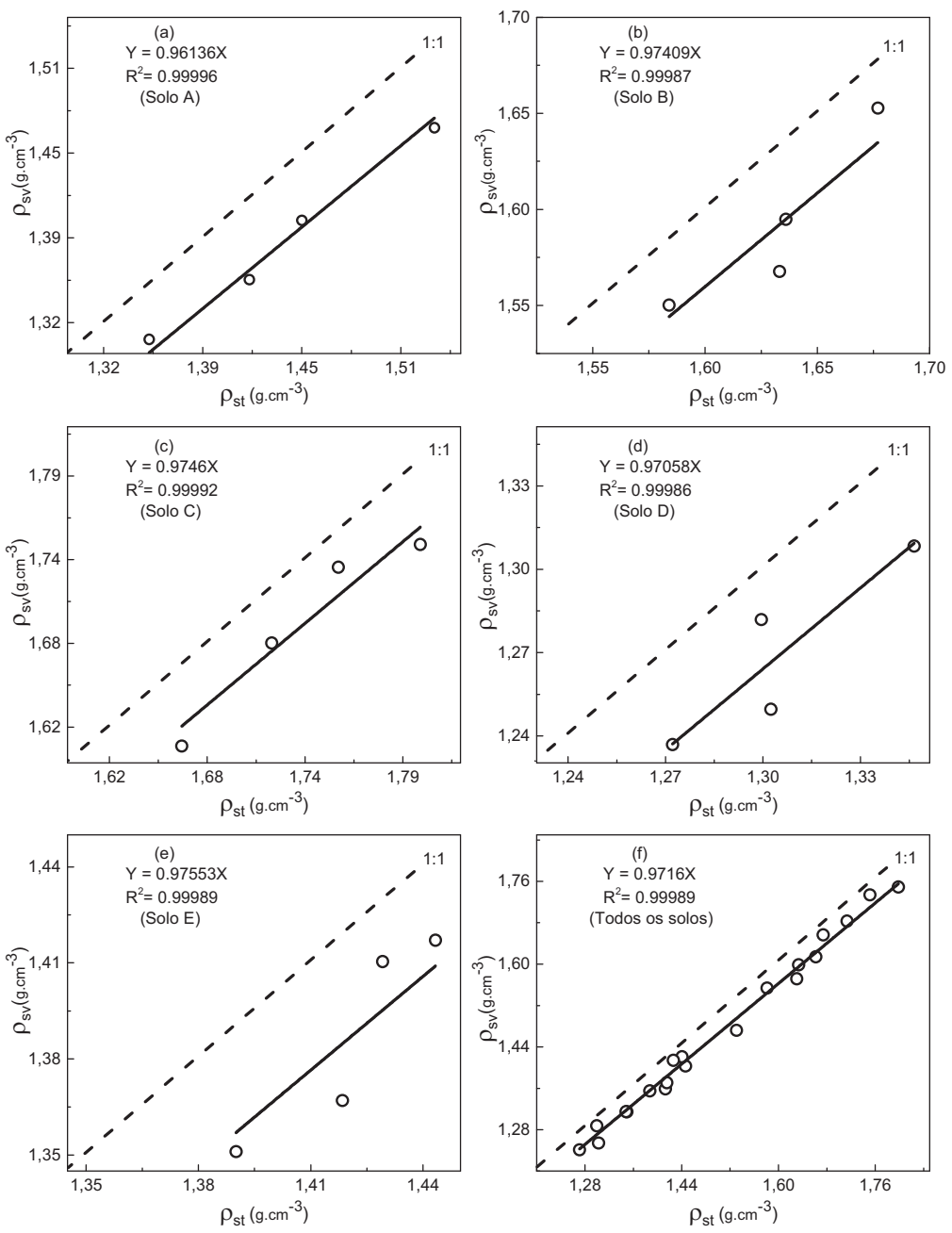

Figura 3: Comparação entre a densidade do solo obtida pelo método do torrão parafinado, $\rho_{s t}$, e pelo método da sela a vácuo, $\rho_{s v}$, para os diferentes tipos de solos. Os pontos representados por círculos vazados indicam os valores medidos de $\rho_{s t}$ e $\rho_{s v}$ para uma mesma amostra de solo, as linhas cheias indicam a reta de ajuste dos pontos e as linhas tracejadas indicam valores iguais de $\rho_{s t} \mathrm{e}$ $\rho_{s v}$ e servem como referência para a comparação (reta 1:1). Em (a) e (b) Latossolo Vermelho-Amarelo, em (c) Nitossolo Vermelho, em (d) Latossolo Bruno e em (e) Cambissolo Háplico Alumínico. Em (f) comparação das densidades do solo para os cinco conjuntos de solos analisados neste trabalho.

os solos analisados independentemente das diferenças observadas na textura.

O trabalho de [1] investigou a eficácia da impermeabilização com parafina de amostras usadas na avaliação de $\rho_{s}$. Estes autores utilizaram a técnica de microtomografia de raios $\mathrm{X}$ para verificar a estrutura interna dos agregados e averiguar a possível entrada de parafina nas amostras de solo. Foi observado que as amostras com entrada de parafina apresentaram valores maiores de $\rho_{s}$ induzidos pelo procedimento de impermeabilização. Os autores associam tal resultado à redução do volume medido do solo devido à entrada da parafina no interior dos agregados, fato comprovado pelas imagens tridimensionais apresentadas no trabalho.

Outros trabalhos também relatam maiores valores de $\rho_{s}$ medidos pelo método do torrão parafinado em comparação com outros métodos (anel volumétrico, sonda de nêutrons-gama, escavação), e, apontam como causas, além da redução do volume medido do solo devido ao possível preenchimento de seus poros com a parafina, o fato dos agregados coletados geralmente serem mais densos do que as amostras usadas nos outros métodos 6, 16. Outro fator indicado nestes trabalhos como provável fonte dessa superestimação no valor da $\rho_{s}$ é a perda de informação causada pela seleção de amostras, pois são desconsiderados os poros interagregados, fissuras e outros elementos que têm relação direta com a $\rho_{s}[10,17,18$.

No método da sela a vácuo a entrada de matéria no interior da amostra é improvável, pois a embalagem plástica, ao ser evacuada, assume a forma externa do agregado de solo. Diferentemente do processo que ocorre com a parafina fundida, não há qualquer tipo de fluido escoando para dentro dos macroporos ou fissuras dos agregados com possibilidade de ocupar os espaços porosos das amostras [11. Na verdade, o que se veri- 
Tabela 2: Valores da densidade do solo medido pelos métodos do torrão parafinado, $\rho_{s t}$, e da sela a vácuo, $\rho_{s v}$. Os valores da densidade do solo são expressos pela média \pm desvio padrão.

\begin{tabular}{lccccc}
\hline & Solo A & Solo B & Solo C & Solo D & Solo E \\
\hline $\boldsymbol{\rho}_{\boldsymbol{s t}}\left(\boldsymbol{g} \cdot \mathbf{c m}^{-\mathbf{3}}\right)$ & $1,44 \pm 0,08$ & $1,63 \pm 0,04$ & $1,74 \pm 0,06$ & $1,31 \pm 0,03$ & $1,42 \pm 0,02$ \\
$\left.\boldsymbol{\rho}_{\boldsymbol{s v} \boldsymbol{( g . c m}} \boldsymbol{( g . 3}^{\mathbf{3}}\right)$ & $1,38 \pm 0,07$ & $1,59 \pm 0,04$ & $1,69 \pm 0,06$ & $1,27 \pm 0,03$ & $1,39 \pm 0,03$ \\
\hline
\end{tabular}

fica é um fluxo de ar saindo da amostra por ação da bomba de vácuo da máquina seladora, com a consequente atuação da pressão atmosférica no entorno da embalagem plástica moldando-a a forma da amostra de solo.

Na Tabela 2 apresentam-se os valores médios da $\rho_{s t}$ e $\rho_{s v}$ para todos os solos analisados. A $\rho_{s v}$ foi menor que a $\rho_{s t}$ em todos os casos. Os dados observados no presente estudo corroboram com a característica supracitada do método da sela a vácuo, ou seja, valores menores para a $\rho_{s}$ medidos por este método em relação ao utilizado como referência; uma vez que no primeiro não há o preenchimento dos poros da amostra, como pode ocorrer com o método do torrão parafinado. Porém, analisando os valores médios e os respectivos desvios padrão é possível observar que existe concordância entre os métodos. Assim, os métodos utilizados neste estudo se mostraram equivalentes e, para os solos analisados, o método da sela a vácuo apresenta indícios de uma melhor determinação do volume da amostra e, consequentemente, nos valores de $\rho_{s}$.

Outra característica do método da sela a vácuo é o fato de ser aparentemente não destrutivo possibilitando a utilização dos agregados para outros estudos, pois o processo de impermeabilização é reversível com mínima perturbação na estrutura da amostra, diferentemente do que ocorre com o torrão parafinado. A maior variação de massa apresentada (diferença entre a $m_{s}$ antes e depois da impermeabilização pelo método da sela a vácuo), dentre todas as amostras analisadas, foi de 1,07 g (variação de $1,37 \%$ na massa total da amostra) e essa perda de massa pela amostra em questão se deve a pequena compressão que a mesma sofreu no processo de evacuação do ar com consequente desagregação de partículas localizadas na superfície da amostra. Considerando todas as 20 amostras de solo, a perda média de massa foi de $0,32 \mathrm{~g}$, o que indica a baixa perturbação nas amostras.

A Figura 4 apresenta a variação média da massa para os diferentes solos analisados. Embora o solo D apresente uma variação média de massa que destoa dos demais, considerando a massa das amostras deste solo (massa média de 60,08 g), essa variação de massa é considerada desprezível.

Por fim, o método além de ser mais rápido também permite uma maior confiabilidade na impermeabilização das amostras em relação à parafina fundida, pois muitas vezes o torrão impermeabilizado com a parafina precisa ser descartado devido a entrada de água na imersão [19]. Este inconveniente pode ser minimizado com uma maior atenção no momento da imersão da amostra na parafina

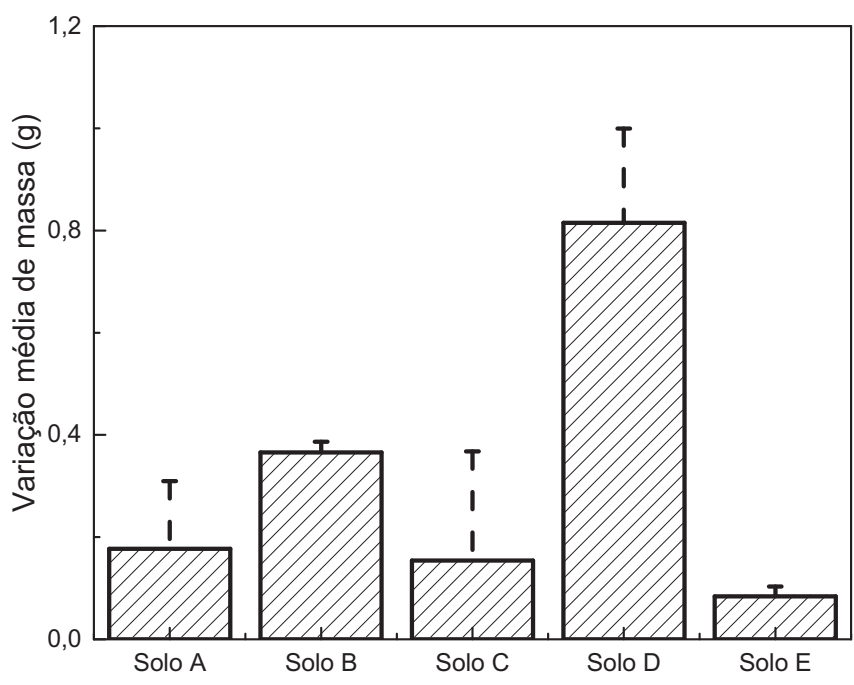

Figura 4: Variação média da massa (média da diferença entre a massa do solo antes e depois da impermeabilização pelo método da sela a vácuo) para os cinco conjuntos de solos. Os solos $A$ e B são Latossolos Vermelho-Amarelo, o solo C é um Nitossolo Vermelho, o solo D é um Latossolo Bruno e o solo E é um Cambissolo Háplico Alumínico.

fundida e principalmente após a solidificação da camada impermeável, buscando por possíveis pontos de entrada de água na camada (orifícios, trincas, bolhas de ar, etc). No entanto, este procedimento aumenta o tempo de preparo das amostras, requer experiência por parte do operador e apenas no momento da imersão em água é que se tem real conhecimento se a camada foi impermeabilizada satisfatoriamente. Em contrapartida, na impermeabilização com sela a vácuo, caso haja pontos falhos na camada impermeável serão facilmente identificados, uma vez que a embalagem plástica não irá reter a evacuação feita pela bomba de vácuo.

\section{Conclusão}

O método da sela a vácuo proposto neste trabalho mostrou-se ser mais rápido no preparo das amostras e com maior confiabilidade na impermeabilização quando comparado ao método do torrão parafinado, resultando em otimização de tempo e reduzindo a incidência de perda de amostra por entrada de água no momento das medidas. A metodologia alternativa apresentou boa concordância com o método do torrão utilizado como referência. Ressalta-se ainda a característica reversível da impermeabilização com o método da sela a vácuo, com baixa perturbação na amostra. Esta característica 
permitiu reutilizar as mesmas amostras para efetuar novas medidas (densidade do solo pelo método do torrão parafinado) diminuindo as questões relacionadas à variabilidade espacial do solo.

Os resultados também mostram que o método da sela a vácuo, além de confiável para medidas da densidade do solo, pode ser facilmente implementado em atividades laboratoriais voltadas para o ensino de física aplicadas em diferentes áreas de conhecimento. Conceitos como massa, volume, massa específica, densidade, peso real, peso aparente e empuxo podem ser facilmente trabalhados adotando-se o método proposto. Assim, este método mostra-se potencialmente útil para ser utilizado em aulas de física experimental dedicadas: ao ensino médio, mais especificamente o ensino técnico, e aos cursos de ensino superior em que os meios porosos são importantes, principalmente aqueles da área ambiental, licenciatura e bacharelado em física, engenharias e agronomia.

\section{Referências}

[1] J. Van Lier, Fúsica do solo (Sociedade Brasileira de Ciência do Solo, Viçosa, 2010).

[2] W. Hu, M.A. Shao e B.C. Si. European Journal of Soil Science 63, 820 (2012)

[3] T.R. Ferreira, J.A.R. Borges e L.F. Pires. Soil and Tillage Research 152, 74 (2015).

[4] E.K.A. Twum e S. Nii-Annang. Applied and Environmental Soil Science 2015, 1 (2015).

[5] A. Ola, S. Schmidt e C.E. Lovelock. Plant and Soil 432, $91(2018)$

[6] L.C. Timm, L.F. Pires, K. Reichardt, R. Roveratti, J.C.M. Oliveira e O.O.S. Bacchi. Australian Journal of Soil Research 43, 97 (2005).
[7] A.A.G. Al-Shammary, A.Z. Kouzani, A. Kaynak, S.Y. Khoo, M. Norton e W. Gates. Pedosphere 28, 581 (2018).

[8] P.C. Teixeira, G.K. Donagemma, A. Fontana, W.G. Teixeira, Manual de métodos de análise de solo. (Embrapa Solos, Rio de Janeiro, 2017).

[9] A.C. Auler, L.F. Pires, A.M. Brinatti e S.C. Saab. Revista Brasileira de Ensino de Física 39, e1506 (2017).

[10] A. Solgi, R. Naghdi, E.R. Labelle, P.A. Tsioras e A. Salehi. Croatian Journal of Forest Engineering 39, 247 (2018).

[11] L.F. Pires, S.J. Mooney, A.C. Auler, B. Atkinson e C.J. Sturrock. Geoderma 337, 935 (2019).

[12] H.G. Santos, P.K.T. Jacomine, L.H.C. Anjos, V.A. Oliveira, J.F. Lumbreras, M.R. Coelho, J.A. Almeida, J.C.A. Filho, J.B. Oliveira e T.J.F. Cunha. Sistema Brasileiro de Classificação de Solos (Embrapa Solos, Rio de Janeiro, 2018).

[13] N.J. Mckenzie, K. Coughlan e H.P. Cresswell. Soil physical measurement and interpretation for land evaluation (Csiro Publishing, Melbourne, 2002).

[14] D. Hillel. Environmental soil physics: Fundamentals, applications, and environmental considerations (Academic Press, San Diego, 1998).

[15] L.F. Pires, A.M. Brinatti, S.C. Saab e F.A.M. Cássaro. Applied Radiation and Isotopes 92, 37 (2014).

[16] L.F. Pires, J.A. Rosa e L.C. Timm. Acta Scientiarum Agronomy 33, 161 (2011).

[17] V.R. Silva, D.J. Reinert e J.M. Reichert. Ciência Rural 30, 1065 (2000).

[18] M. Casanova, E. Tapia, O. Seguel e O. Salazar. Chilean journal of agricultural research 76, 105 (2016).

[19] G.R. Blake, in Methods of Soil Analysis: Part 1 Physical and Mineralogical Properties, Including Statistics of Measurement and Sampling, editado por C.A. Black (American Society of Agronomy, Madison, 1965). 